\title{
Desempenho de espécies e procedências de Eucalyptus no Planalto Norte Catarinense, Brasil
}

\author{
Performance of Eucalyptus species and provenances in the Northern \\ Plateau of Santa Catarina, Brazil
}

Taciana Frigotto $^{1}$ (D), Marcio Carlos Navroski ${ }^{1}$ (D), Natália Saudade de Aguiar $^{2}$ (B), Dionéia Felippe $^{1}$ (D), Geedre Adriano Borsoi ${ }^{1}$ (1), Mariane de Oliveira Pereira ${ }^{1}$ (1), Queli Cristina Lovatel ${ }^{1}$ (1)

${ }^{1}$ Universidade do Estado de Santa Catarina - UDESC, Lages, SC, Brasil

${ }^{2}$ Universidade Federal do Paraná - UFPR, Curitiba, PR, Brasil

Como citar: Frigotto, T., Navroski, M. C., Aguiar, N. S., Felippe, D., Borsoi, G. A., Pereira, M. O., \& Lovatel, Q. C. (2020). Desempenho de espécies e procedências de Eucalyptus no Planalto Norte Catarinense, Brasil. Scientia Forestalis, 48(127), e3273. https://doi.org/10.18671/scifor.v48n127.14

\begin{abstract}
Resumo
O objetivo do estudo foi avaliar espécies e procedências de Eucalyptus spp., selecionando aquelas que apresentam elevada sobrevivência e capacidade produtiva nas condições da região do Planalto Norte Catarinense. O teste está localizado no município de Rio Negrinho, ao norte do Estado de Santa Catarina. O povoamento foi implantado em dezembro de 1999 com treze espécies do gênero Eucalyptus, oriundas de sementes de diferentes procedências. O experimento foi instalado em delineamento em blocos ao acaso, com três repetições e parcelas lineares. As variáveis avaliadas foram sobrevivência (\%), altura (m), diâmetro a altura do peito $(\mathrm{cm})$, volume $\left(\mathrm{m}^{3}\right)$ e bifurcação dos indivíduos. As espécies potenciais em relação às características quantitativas foram E. smithii, E. dunnii, E. benthamii, E. badjensis e E. dorrigoensis. Já para as variáveis qualitativas E. badjensis, E. macarthurii, E. benthamii e E. dorrigoensis apresentaram os melhores resultados. Foi possível verificar a ampla variabilidade das características avaliadas entre procedências, destacando a importância da escolha de materiais genéticos adaptados e com características quantitativas superiores. O estudo demonstra que outras espécies de Eucalyptus, além das usualmente plantadas na região, podem apresentar potencial para povoamentos comerciais, tolerando as baixas temperaturas, a ocorrência de geadas e apresentando boa produtividade.
\end{abstract}

Palavras-chave: Eucalipto; Sobrevivência; Crescimento; Geadas; Espécies potenciais.

\begin{abstract}
The objective of this study was to evaluate species and provenances of Eucalyptus spp., selecting those with high survival and productive potential under the conditions of the Santa Catarina Plateau. The test is located in the municipality of Rio Negrinho, northern Santa Catarina state. The stand was established in December 1999 with thirteen species of the genus Eucalyptus, originating from seeds of different origins. The experiment was carried out in a randomized block design with three replications and linear plots. The variables evaluated were survival $(\%)$, height $(m)$, diameter at breast height $(\mathrm{cm})$, volume $\left(\mathrm{m}^{3}\right)$ and bifurcation of the individuals. Potential species in relation to quantitative characteristics were $E$. smithii, E. dunnii, E. benthamii, E. badjensis and E. dorrigoensis. For the qualitative variables E. badjensis, $E$. macarthurii, E. benthamii and $E$. dorrigoensis presented the best results. It was possible to verify the wide variability of the characteristics evaluated between provenances, highlighting the importance of choosing adapted genetic materials with superior quantitative characteristics. The study shows that other Eucalyptus species, besides those usually planted in the region, have a potential for commercial stands, tolerating low temperatures, frost occurrence and good productivity.
\end{abstract}

Keywords: Eucalypts; Survival; Growth; Frost; Potential species.

Fonte de financiamento: FAPESC - grupo de pesquisa Gestão de Recursos Florestais (PAP2019031000055); Coordenação de Aperfeiçoamento de Pessoal de Nível Superior (CAPES) código 001; Empresa Souza Cruz ${ }^{\circledR}$.

Conflito de interesse: Nada a declarar.

Autor correspondente: marcio.navroski@udesc.br

Recebido: 4 fevereiro 2019.

Aceito: 3 setembro 2019.

Editor: Paulo Henrique Müller Silva.

(c) (i) Este é um artigo publicado em acesso aberto (Open Access) sob a licença Creative Commons Attribution, que permite uso, distribuição e reprodução em qualquer meio, sem restrições desde que o trabalho original seja corretamente citado. 


\section{INTRODUÇÃO}

O gênero Eucalyptus pertence à família Myrtaceae e possui cerca de 600 espécies e subespécies, quase todas nativas da Austrália. O gênero se destaca pelas características de rápido crescimento, alta produtividade, ampla diversidade de espécies, grande capacidade de adaptação e aplicação para diversas finalidades, sendo amplamente utilizado para plantios florestais comerciais (Correia, 2011).

Os plantios de eucalipto ocupam no Brasil atualmente uma área superior a 5,7 milhões de hectares. O país se destaca pela produtividade florestal, sendo que em 2016 liderou o ranking global com uma média de $35,7 \mathrm{~m}^{3} \mathrm{ha}^{-1} \mathrm{ano}^{-1}$ para os plantios de eucalipto (Indústria Brasileira de Árvores, 2017). Ao longo das últimas décadas se verificaram crescentes aumentos na produtividade das florestas de eucalipto, graças ao desenvolvimento de materiais genéticos de maior potencial produtivo, sobretudo pelo aprimoramento da hibridação e clonagem, bem como a evolução das técnicas de manejo florestal (Assis, 2014).

O gênero Eucalyptus apresenta ampla plasticidade e dispersão mundial, crescendo satisfatoriamente em diferentes situações edafoclimáticas, extrapolando aquelas das regiões de origem (Santos et al., 2001). O Sul do Brasil abrange áreas com climas subtropicais, com regularidade de chuvas ao longo do ano, enquadrados como Cfa (Clima subtropical, com verão quente) e Cfb (Clima temperado, com verão ameno), de acordo com a classificação de Köppen (Alvares et al., 2013). As áreas elevadas dos estados de Santa Catarina, Rio Grande do Sul e Paraná apresentam temperaturas relativamente baixas e número considerável de geadas, que afetam o cultivo de eucaliptos com baixa tolerância ao frio (Konzen et al., 2017).

Dessa forma, apesar do grande potencial florestal da região, a expansão de povoamentos do gênero Eucalyptus ainda tem ocorrido de forma limitada, principalmente devido à ocorrência de geadas e ao reduzido número de espécies que se adaptam a esta condição climática (Müller et al., 2017). As espécies deste gênero mais cultivadas atualmente nesses locais são Eucalyptus benthamii Maiden et Cambage e Eucalyptus dunnii Maiden. Contudo, outras espécies têm apresentado desenvolvimento silvicultural satisfatório em plantios experimentais, que podem vir a ampliar ou substituir esse número limitado de espécies (Brondani et al., 2010). A introdução de espécies com tolerância às baixas temperaturas ou mesmo híbridos com espécies alternativas é importante para aumentar a produção de eucaliptos em regiões mais frias (Konzen et al., 2017).

De acordo com Paludzyszyn Filho et al. (2006), o sucesso ou insucesso do plantio pode advir da escolha da espécie, da região de procedência geográfica e da região de procedência das sementes. Sabe-se que o comportamento das espécies frequentemente é inesperado quando introduzidas em novos locais (Ferreira \& Couto, 1981). Assim, para a escolha das espécies que irão compor uma floresta com vistas a produção é imprescindível a realização de testes de espécies para avaliação da sua capacidade de aclimatação em cada local (Quiqui et al., 2001).

As variações genéticas existentes entre procedências dentro de uma mesma espécie surgem como resultado da adaptação das espécies às condições edafoclimáticas dos habitats (Kageyama et al., 1977). Assim, o comportamento de diferentes procedências de eucaliptos, quando submetidas a fatores ambientais diversos, também deve ser avaliado, certificando-se de que os plantios obterão êxito (Higa \& Higa, 2000).

Diante do exposto, o objetivo do estudo foi avaliar espécies e procedências de Eucalyptus spp. na região do Planalto Norte Catarinense, selecionando aquelas que apresentam elevada sobrevivência e capacidade produtiva nestas condições.

\section{MATERIAL E MÉTODOS}

O povoamento de Eucalyptus spp. no qual foi realizado o estudo está localizado em Rio Negrinho, SC, Brasil. O município possui altitude próxima a 790 metros e solos predominantemente das classes Cambissolo Háplico Distrófico e Neossolo Regolítico Distrófico (Kobiyama et al., 2004). 
A região do Planalto Norte Catarinense, onde se localiza o teste, é considerada de clima Cfb (clima temperado, subtropical úmido, com regularidade das chuvas ao longo de todo o ano) pela classificação de Köppen (Alvares et al., 2013). A temperatura média anual do município é de $16,8^{\circ} \mathrm{C}$, com temperatura média mínima anual de $13^{\circ} \mathrm{C}$ e máxima de $22,8^{\circ} \mathrm{C}$ (Wrege et al., 2012). A precipitação pluviométrica total anual média é $1720 \mathrm{~mm}$ (Empresa de Pesquisa Agropecuária e Extensão Rural de Santa Catarina, 2009).

Na estação meteorológica de Rio Negrinho o número médio de geadas anuais foi de 10,21 entre os anos de 1990 e 2003; em outro município do Planalto Norte Catarinense, Matos Costa, o número médio de geadas anuais foi 18,5. Os episódios de geadas no Estado de Santa Catarina são mais frequentes entre os meses de maio e setembro, principalmente em junho e julho (Aguiar \& Mendonça, 2004).

O talhão com as diferentes espécies/procedências foi plantado em dezembro de 1999, em espaçamento $3 \times 2$ metros. As treze espécies do gênero Eucalyptus sp. avaliadas são de origem seminal, provenientes de diversos locais, conforme demonstrado na Tabela 1.

Tabela 1 - Espécies e procedências de Eucalyptus implantadas em 1999 no município de Rio Negrinho, SC.

\begin{tabular}{|c|c|c|}
\hline Espécie & Procedência das sementes & País \\
\hline E. smithii & Papel e Celulose Catarinense (PCC-SC) & Brasil \\
\hline E. smithii & Taboaganda & Austrália \\
\hline E. smithii & Austrália & Austrália \\
\hline E. smithii & Wilsons Promontory & Austrália \\
\hline E. nitens & Taboaganda & Austrália \\
\hline E. nitens & Austrália & Austrália \\
\hline E. nitens & Macalista & Austrália \\
\hline E. nitens & Glen Boc & Austrália \\
\hline E. benthamii & Papel e Celulose Catarinense (PCC-SC) & Brasil \\
\hline E. benthamii & Austrália & Austrália \\
\hline E. dorrigoensis & Austrália & Austrália \\
\hline E. macarthurii & Wilsons Promontory & Austrália \\
\hline E. macarthurii & Austrália & Austrália \\
\hline E. macarthurii & Pieter Maritz & Austrália \\
\hline E. badgensis & Glen Boc & Austrália \\
\hline E. badgensis $x$ nitens & Glen Boc & Austrália \\
\hline E. deanei & Papel e Celulose Catarinense (PCC-SC) & Brasil \\
\hline E. deanei & Klabin/PR & Brasil \\
\hline E. deanei & Manville Produtos Florestais/SC & Brasil \\
\hline E. viminalis & Nacional Campo & Brasil \\
\hline E. viminalis & Bonani & Brasil \\
\hline E. viminalis & Manville Produtos Florestais/SC & Brasil \\
\hline E. viminalis & BGNDO & Brasil \\
\hline E. viminalis & Canela/RS & Brasil \\
\hline E. obliqua & Riocell - Celulose Riograndense/RS & Brasil \\
\hline E. dunnii & Acacia Creek N. Lgguns & Austrália \\
\hline E. grandis & Lajeado/RS & Brasil \\
\hline
\end{tabular}

O experimento foi instalado com delineamento em blocos ao acaso, de três repetições e parcelas lineares. Cada linha de plantio foi composta por uma espécie e/ou procedência, com 45 árvores por linha. Nos locais em que houve a mortalidade de indivíduos foi realizado o replantio com mudas de E. dunnii, devido à dificuldade de encontrar mudas do mesmo material genético. As mudas replantadas não foram avaliadas. 
A avaliação do experimento foi realizada em 2015, sendo consideradas as seguintes variáveis: sobrevivência - SOB (\%), altura - $H(m)$, diâmetro na altura do peito - DAP $(\mathrm{cm})$, volume - VOL $\left(\mathrm{m}^{3}\right.$ ind $\left.^{-1}\right)$ e bifurcação - BIF (\%), aos 16 anos. A avaliação da bifurcação foi realizada como critério binomial (0 - não bifurcado e 1 - bifurcado). A obtenção do volume individual foi realizada selecionando 20 árvores-amostra por espécie/procedência, contemplando todas as classes diamétricas. Por se tratar de uma área experimental com vistas para a seleção de material superior, foi considerada inviável a utilização de métodos destrutivos para obter o volume individual. Portanto, foi realizada a cubagem pelo método de Smalian, sendo utilizado o dendrômetro Criterion RD1000.

Para estimativa do volume individual foram testados doze modelos, conforme trabalho de Frigotto et al. (2015) realizado com estes dados. Com base nos resultados apresentados na análise gráfica de resíduos, erro padrão da estimativa $\left(S_{y x}\right)$ e coeficiente de determinação

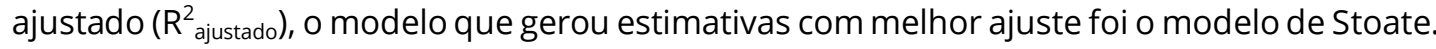
A equação ajustada foi $v=0,08357-0,0002 . d a p^{2}+0,0000404\left(\operatorname{dap}^{2} . h\right)-0,0028 . h+\varepsilon_{i}$, em que: v= volume; dap= diâmetro na altura do peito; $\mathrm{h}=$ altura total; $\varepsilon_{i}=$ erro absoluto. A equação apresentou

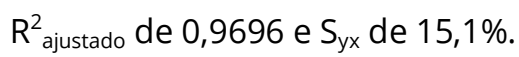

A normalidade do conjunto de dados foi confirmada por meio do teste de KolmogorovSmirnov e a homogeneidade pelo teste de Bartlett. A análise de variância (ANOVA) foi realizada e as médias foram comparadas pelo teste de Scott-Knott a 5\% de probabilidade de erro.

\section{Estimativa dos componentes genéticos}

As estimativas dos parâmetros genéticos e fenotípicos foram efetuadas através do programa genético-estatístico Selegen-Reml/Blup (Resende, 2006). Utilizou-se o modelo 24: blocos ao acaso, teste de populações ou procedências, várias plantas por parcela, em que $y=$ $\mathrm{Xr}+\mathrm{Zg}+\mathrm{Wp}+\mathrm{e}$, em que y é o vetor de dados, $\mathrm{r}$ é o vetor dos efeitos de repetição (assumidos como fixos) somados à média geral, g é o vetor dos efeitos genotípicos de populações (assumidos como aleatórios), p é o vetor dos efeitos de parcela, e é o vetor de erros ou resíduos (aleatórios).

$A$ análise gerou estimativas dos seguintes parâmetros genéticos: $V_{g}$ : variância genotípica entre populações ou procedências; $V_{\text {parc }}$ : variância ambiental entre parcelas; $V_{\mathrm{e}}$ : variância residual dentro de parcela; $\mathrm{V}_{\mathrm{f}}$ : variância fenotípica individual; $\mathrm{h}_{\mathrm{g}}^{2}$ : herdabilidade individual no sentido amplo; $\mathrm{C}_{\text {parc: }}^{2}$ coeficiente de determinação dos efeitos da parcela; $\mathrm{h}^{2}{ }_{\mathrm{mp}}$ : herdabilidade da média da procedência, assumindo estande completo; $A c_{\text {proc: }}$ Acurácia da seleção de procedências, assumindo estande completo. A utilização do método REML para estimar os componentes da variância, deve-se ao desbalanceamento experimental em termos do número desigual de árvores sobreviventes por parcelas.

\section{RESULTADOS E DISCUSSÃO}

\section{Seleção de espécies}

A análise de variância revelou diferenças significativas $(p<0,005)$ para todas as variáveis avaliadas, mostrando potencial para seleção de espécies e entre essas, das melhores procedências.

Para a variável quantitativa diâmetro na altura do peito (DAP) o conjunto de espécies composta por Eucalyptus smithii R.T. Baker, Eucalyptus dunnii Maiden, Eucalyptus benthamii Maiden \& Cambage, Eucalyptus badjensis Beuzev. \& Welch, Eucalyptus dorrigoensis (Blakely) L.A.S. Johnson \& K.D. Hill, Eucalyptus deanei Maiden e o híbrido Eucalyptus badjensis Beuzev. \& Welch $x$ Eucalyptus nitens Maiden apresentaram os melhores resultados, não diferindo estatisticamente entre si. Estas mesmas espécies, com exceção de $E$. deanei e o híbrido $E$. badjensis $x$ E. nitens também apresentaram os maiores valores de altura e volume individual (Tabela 2). 
Tabela 2 - Média das variáveis diâmetro na altura do peito - DAP $(\mathrm{cm})$, altura - H $(\mathrm{m})$, volume - VOL $\left(\mathrm{m}^{3}\right.$ ind $^{-1}$ ), sobrevivência - SOB (\%) e bifurcação do fuste - BIF (\%) para as treze espécies de Eucalyptus aos 16 anos de idade.

\begin{tabular}{cccccc}
\hline Espécie & DAP $(\mathbf{c m})$ & $\mathbf{H}(\mathbf{m})$ & VOL $\left(\mathbf{m}^{3}\right.$ ind $\left.^{-1}\right)$ & SOB $(\%)$ & BIF $(\%)$ \\
\hline E. smithii & $30,7 \mathrm{a}^{*}$ & $37,0 \mathrm{a}$ & $1,9195 \mathrm{a}$ & $32 \mathrm{c}$ & $11 \mathrm{~b}$ \\
E. dunnii & $30,0 \mathrm{a}$ & $36,9 \mathrm{a}$ & $1,9094 \mathrm{a}$ & $83 \mathrm{a}$ & $16 \mathrm{a}$ \\
E. benthamii & $29,7 \mathrm{a}$ & $36,5 \mathrm{a}$ & $1,8831 \mathrm{a}$ & $55 \mathrm{~b}$ & $1 \mathrm{~b}$ \\
E. badjensis & $29,0 \mathrm{a}$ & $36,0 \mathrm{a}$ & $1,8544 \mathrm{a}$ & $42 \mathrm{~b}$ & $5 \mathrm{~b}$ \\
E. dorrigoensis & $25,4 \mathrm{a}$ & $35,6 \mathrm{a}$ & $1,8262 \mathrm{a}$ & $59 \mathrm{~b}$ & $2 \mathrm{~b}$ \\
E. deanei & $23,5 \mathrm{a}$ & $32,9 \mathrm{~b}$ & $1,6588 \mathrm{~b}$ & $55 \mathrm{~b}$ & $18 \mathrm{a}$ \\
E. badjensis X E. nitens & $23,4 \mathrm{a}$ & $32,8 \mathrm{~b}$ & $1,6476 \mathrm{~b}$ & $32 \mathrm{c}$ & $23 \mathrm{a}$ \\
E. viminalis & $22,4 \mathrm{~b}$ & $32,8 \mathrm{~b}$ & $1,6462 \mathrm{~b}$ & $34 \mathrm{c}$ & $10 \mathrm{~b}$ \\
E. macarthurii & $21,1 \mathrm{~b}$ & $31,6 \mathrm{~b}$ & $1,5690 \mathrm{~b}$ & $54 \mathrm{~b}$ & $5 \mathrm{~b}$ \\
E. dalrympleana & $21,0 \mathrm{~b}$ & $31,4 \mathrm{~b}$ & $1,5652 \mathrm{~b}$ & $10 \mathrm{~d}$ & $37 \mathrm{a}$ \\
E. grandis & $19,2 \mathrm{c}$ & $31,0 \mathrm{~b}$ & $1,5360 \mathrm{~b}$ & $47 \mathrm{~b}$ & $13 \mathrm{a}$ \\
E. nitens & $18,1 \mathrm{c}$ & $29,6 \mathrm{c}$ & $1,4425 \mathrm{~b}$ & $24 \mathrm{c}$ & $10 \mathrm{~b}$ \\
E. obliqua & $13,6 \mathrm{c}$ & $27,7 \mathrm{c}$ & $1,3222 \mathrm{~b}$ & $13 \mathrm{~d}$ & $20 \mathrm{a}$ \\
\hline
\end{tabular}

*Médias na mesma coluna seguidas por letras distintas diferem entre si pelo teste de Scott-Knott a $P<0,05$.

E. smithii apresentou os melhores resultados para todas as variáveis dendrométricas $\left(\mathrm{DAP}=30,7 \mathrm{~cm} ; \mathrm{H}=37,0 \mathrm{~m} ; \mathrm{VOL}=1,9195 \mathrm{~m}^{3}\right.$ ). Entretanto, em relação à sobrevivência (SOB) a espécie não obteve bons resultados, apresentando somente $32 \%$ aos 16 anos. Higa et al. (1997) em região fria do estado do Paraná encontrou valores semelhantes para a sobrevivência, sendo inferior a 25\%. Para Yi et al. (2014) em uma região fria da China, encontraram $57 \%$ dos indivíduos de $E$. smithii com danos severos e extremamente severos devido ao congelamento, demonstrando a baixa resistência ao frio extremo.

Entretanto, pode-se dizer que apesar da alta mortalidade, os indivíduos remanescentes se recuperaram dos danos sofridos e apresentaram excelente desenvolvimento. A espécie é conhecida em algumas regiões da China pela alta taxa de crescimento, boa forma do caule, tolerância relativa ao frio e alto rendimento de óleos essenciais nas folhas (Chen, 2002; Zhang et al., 2003). Pesquisas na África do Sul também mostraram que a espécie tem rápido crescimento inicial, é moderadamente resistente ao congelamento e a seca, sendo plantada em grande escala em algumas áreas mais frias da África do Sul para a produção de óleos essenciais e madeira para celulose em rotação curta (Swain \& Gardner, 2003). Em estudo realizado na China por Arnold et al. (2004), E. smithii se destacou no crescimento em diâmetro e altura, sendo recomendada para o plantio.

A espécie $E$. dunnii é uma das espécies de maior crescimento dentre os eucaliptos, sobretudo dentro da secção Maidenaria (Fonseca et al., 2010). Ao analisar a espécie, nota-se que a mesma obteve bons resultados para as variáveis dendrométricas. A espécie apresenta alto potencial de produção em área de plantio em clima temperado, com potencial de produção de até $50 \mathrm{~m}^{3}$ ha $^{-1}$ ano-1 de madeira para múltiplos usos. Vale ressaltar que $E$. dunnii pode apresentar crescimento inicial semelhante ao E. grandis em climas tendendo a tropical. Após esse período, há tendência de declínio do crescimento relativo se não houver compensações de retenção de umidade no solo (Paludzyszyn Filho et al., 2006). Higa et al. (1997) enquadrou $E$. dunnii no grupo que apresentou maior crescimento em experimento no Paraná.

E. benthamii também faz parte do grupo de espécies que se destacou nas caraterísticas quantitativas. No Sul do Brasil, E. benthamii tem mostrado bom crescimento e resistência a 
geadas em Santa Catarina. A espécie também foi apontada como promissora em áreas montanhosas do Estado de Minas Gerais (Carpanezzi et al., 1988). Testes implantados em Colombo e Guarapuava (PR), indicam alta tolerância a geadas e crescimento médio superior ao E. dunnii (Paludzyszyn Filho et al., 2006). No estudo atual não houve diferença significativa no crescimento em diâmetro, altura e volume entre as duas espécies.

Em geral, E. badjensis surge como uma boa alternativa para o cultivo em área frias, com crescimento compatível a de outras espécies temperadas, tolerância a temperaturas abaixo de $0^{\circ} \mathrm{C}$ e apresenta mortalidade intermediária (Paludzyszyn Filho \& Santos, 2011), fato que ocorreu nesse estudo. Em experimento instalado no Paraná, E. badjensis se apresentou como uma espécie de rápido crescimento, sendo considerada como uma espécie altamente potencial para plantios no Sul do Brasil em função da alta taxa de crescimento e resistência a geadas (Higa et al., 2002).

E. dorrigoensis faz parte do grupo de espécies que obteve melhores resultados para as variáveis dendrométricas e também se destacou para as variáveis qualitativas. Em um estudo comparando diferentes espécies de Eucalyptus em região fria da China foi observado que $E$. dorrigoensis juntamente com E. benthamii e E. dunnii apresentaram comportamento semelhantes para altura e diâmetro, aos 6 anos (Arnold et al., 2015), resultado semelhante ao encontrado no presente experimento, avaliado aos 16 anos de idade.

A espécie $E$. deanei e o híbrido $E$. badjensis $x$ E. nitens apresentaram boas médias de DAP, porém, em relação à altura obtiveram médias menores, e consequentemente, menor volume médio individual. O híbrido $E$. badjensis $x$ E. nitens ficou em posição intermediária entre as duas espécies nas variáveis quantitativas; assim, pode-se dizer que a hibridização com $E$. nitens, produziu plantas com menores dimensões.

Com relação à sobrevivência a média geral foi de $40 \%$, variando de $10 \%$ a $83 \%$ para $E$. dalrympleana e E. dunnii, respectivamente. Como a área do estudo não apresentou avaliações nos primeiros anos após a implantação, não é possível determinar qual a causa exata da mortalidade das plantas. Contudo, por se tratar de um teste de espécies, no qual foi realizado o plantio de todos os indivíduos no mesmo momento, é possível supor que a principal causa de mortalidade seja o clima da região, especialmente a ocorrência de geadas. De acordo com Higa et al. (1997) um número reduzido de espécies do gênero Eucalyptus é indicado para plantios na região Sul do Brasil, onde as geadas são severas. Devido a essa condição determinante, a seleção de espécies e procedências de Eucalyptus resistentes ao frio é a primeira fase dos programas de melhoramento genéticos dirigidos ao aumento da resistência a geadas.

De acordo com Aguiar \& Mendonça (2004), compilando dados de 33 estações meteorológicas do Estado de Santa Catarina, o ano de 1999 foi o que apresentou o maior número de episódios de geadas nos 24 anos de registros analisados. Já no ano de 2000 ocorreram as geadas mais intensas, principalmente no mês de julho. Coincidentemente, esses foram os anos dois primeiros anos após a instalação do teste, o que pode ter causado efeito direto na sobrevivência dos indivíduos.

A sobrevivência e a porcentagem de árvores bifurcadas podem ser utilizadas como indicadores da adaptabilidade às condições do local de plantio, especialmente em áreas com ocorrência de geadas (Higa et al., 1997). A amplitude de sobrevivência das espécies demonstra como o gênero Eucalyptus se adaptada a diferentes habitats e respondem às mesmas condições ambientais diferentemente.

As espécies que apresentaram as maiores taxas de sobrevivência foram: $E$. dunnii (superior às demais), E. dorrigoensis, E. benthamii, E. deanei, E. badjensis, Eucalyptus macarthurii H. Deane \& Maiden, e Eucalyptus grandis W. Mill ex Maiden; todas com sobrevivência superior à média geral de $40 \%$. Dentro deste grupo destaca-se as espécies que apresentaram menor taxa de bifurcação, inferior a 5\% (E. badjensis, E. macarthurii, E. benthamii e E. dorrigoensis) demonstrando a adaptação às condições ambientais locais, com poucas árvores danificadas.

Entre as espécies citadas no grupo que se destacou nas variáveis qualitativas, E. badjensis obteve uma taxa relativamente alta de sobrevivência (42\%) assim como baixo índice de bifurcação (5,6\%). Em geral, E. badjensis aparece como uma boa alternativa para o cultivo em 
área frias, com crescimento compatível a de outras espécies temperadas, tolerância a temperaturas abaixo de $0^{\circ} \mathrm{C}$ e apresenta mortalidade intermediária (Paludzyszyn Filho \& Santos, 2011), assim como no presente estudo.

E. macarthurrii possui aptidão em resistir ao frio, confirmada pela satisfatória sobrevivência obtida (54\%). Higa et al. (1997) já havia recomendado a espécie em estudo realizado em Campo do Tenente (PR), região fria com ocorrência de geadas, juntamente com E. deanei, que foram consideradas espécies promissoras para a região. Porém, o autor havia encontrado sobrevivência superior a $90 \%$. A maior mortalidade nesse estudo pode ser decorrente de outros fatores, ou a ocorrência de geadas de maior intensidade.

A espécie $E$. benthamii vem sendo indicada para plantios na região Sul do Brasil sujeitas a geadas e temperaturas de até $-6^{\circ} \mathrm{C}$ (Paludzyszyn Filho \& Santos, 2011). Ainda sobre o desenvolvimento do E. benthamii no sul do Brasil, Benin (2014) cita o alto potencial da espécie no estabelecimento de reflorestamentos e suprimento de matéria-prima, devido a adaptação frente às condições climáticas encontradas nessa região. Estes fatos justificam os bons resultados encontrados para as características qualitativas, apresentando $55 \%$ de sobrevivência e apenas $1 \%$ dos indivíduos com bifurcação.

E. dorrigoensis apresentou 59\% de sobrevivência e 2\% de bifurcação. Em um estudo comparando diferentes espécies de Eucalyptus na China, foi observado que $E$. dorrigoensis juntamente com E. benthamii e $E$. dunnii apresentaram comportamento semelhantes para crescimento (altura e diâmetro), no entanto, a sobrevivência apresentou um percentual inferior em relação às outras duas espécies, devido à ocorrência de geadas severas e temperaturas mínimas de até $-8^{\circ} \mathrm{C}$ (Hesheng et al., 2003).

E. dunnii, E. grandis e E. deanei apesar de maiores taxas de sobrevivência (>47\%), apresentaram maior taxa de bifurcação (entre 13 e 18\%), diferindo estatisticamente das demais, efeito que pode ter sido causado por geadas e posterior recuperação das plantas. Entretanto, E. dunnii merece destaque em relação a sobrevivência, com $83 \%$. Esse resultado pode ser justificado pela adaptação da espécie a regiões de clima frio e com presença de geadas, corroborando a indicação da espécie de Carpanezzi et al. (1988) para o plantio no estado de Santa Catarina, em altitudes de até $1000 \mathrm{~m}$.

A espécie (E. dunnii) é indicada para plantios em regiões com temperaturas mínimas absolutas de até $-5^{\circ} \mathrm{C}$ sob condições de aclimatação prévia, suportando até 22 geadas anuais (Food and Agriculture Organization, 1981). Estudos realizados em regiões frias no Brasil e em outros países demonstraram que com temperaturas de até $-7,9^{\circ} \mathrm{C}$ a sobrevivência foi superior a $90 \%$, indicando a alta resistência dessa espécie à baixas temperaturas, mesmo na fase juvenil (Lisbão Junior, 1980; Darrow, 1994; Higa et al., 1997; Mujiu et al., 2003). Porém deve ser considerada a taxa de bifurcação de $16 \%$, dano que pode ter sido causado pela ocorrência de geadas severas. Em estudo realizado por Higa et al. (1997) E. dunnii foi uma das espécies mais danificadas pelas geadas, com $26 \%$ das plantas bifurcadas, mas apresentou a melhor capacidade de recuperação do crescimento.

E. grandis é a espécie de eucalipto mais utilizada em áreas tropicais e de transição para subtropicais no Brasil e em dezenas de outros países. Deve ser plantada em áreas livres de geadas e de períodos de seca muito prolongados; vale ressaltar a alta suscetibilidade ao frio dessa espécie (Paludzyszyn Filho et al., 2006). Apesar da recomendação, considerando o clima da região, a espécie merece destaque por conta das taxas de sobrevivência e de bifurcação satisfatórias, de $47 \%$ e $13 \%$, respectivamente. Esse fato pode ter ocorrido por conta de adaptações das plantas matrizes ao clima da região, pois as sementes são provenientes do Sul do Brasil, gerando indivíduos mais tolerantes ao clima frio. Para comprovar essa hipótese os indivíduos merecem ser testados e propagados, verificando essa hipótese. A taxa de bifurcação sugere que as plantas sofreram danos por geadas, entretanto houve a posterior recuperação e crescimento destas.

E. deanei embora botanicamente semelhante de E. grandis e E. saligna, tem potencial de crescimento inferior a essas duas espécies, porém é mais resistente ao frio (Moura, 2001). Em sua área de distribuição natural suporta temperaturas mínimas entre $0^{\circ} \mathrm{C}$ e $5^{\circ} \mathrm{C}$, geadas com frequência anual entre 0 e 50, ou mais. Na fase juvenil as plantas podem ser danificadas por 
geadas severas, entretanto, apresentam boa capacidade de recuperação. No sul do Brasil, a espécie é considerada tolerante a geadas, após três anos de idade (Carpanezzi et al., 1988). No presente estudo apresentou $55 \%$ de sobrevivência e $18 \%$ de árvores bifurcadas, taxa relativamente alta, que pode ser explicada pelo dano causado por geadas e posterior recuperação.

Esperava-se que as espécies Eucalyptus nitens e Eucalyptus viminalis se destacassem nas características qualitativas, por serem características de regiões frias, apresentando resultados superiores aos encontrados para sobrevivência e bifurcação. Aparentemente as espécies não se adaptaram bem às condições locais ou algum outro fator biótico ou abiótico acarretou na mortalidade dos indivíduos.

\section{Seleção de procedências}

Em relação às variáveis quantitativas somente E. smithii e E. viminalis apresentaram diferenças entre as procedências (Tabela 3). Dentro da espécie E. smithii, que se destacou nas variáveis dendrométricas, a procedência Wilsons Promontory foi superior às demais (DAP= $36,7 \mathrm{~cm} ; \mathrm{H}=39,9 \mathrm{~m} ; \mathrm{VOL}=2,1021 \mathrm{~m}^{3}$ ).

Tabela 3 - Média das variáveis diâmetro na altura do peito - DAP $(\mathrm{cm})$, altura - H $(\mathrm{m})$, volume - VOL $\left(\mathrm{m}^{3}\right.$ ind $^{-1}$ ), sobrevivência - SOB (\%) e bifurcação do fuste - BIF (\%) para diferentes procedências das espécies de Eucalyptus aos 16 anos de idade.

\begin{tabular}{|c|c|c|c|c|c|c|}
\hline Espécie & Procedência & DAP (cm) & $H(m)$ & VOL $\left(m^{3}\right.$ ind $\left.^{-1}\right)$ & SOB (\%) & BIF (\%) \\
\hline \multirow{4}{*}{ E. smithii } & Wilsons Promontory & $36,7 a *$ & 39,9 a & $2,1021 \mathrm{a}$ & $28 \mathrm{~b}$ & $17 a$ \\
\hline & Taboaganda & $28,7 \mathrm{~b}$ & $36,3 b$ & $1,8717 b$ & $21 \mathrm{~b}$ & $15 a$ \\
\hline & $\mathrm{PCC} / \mathrm{SC}$ & 27,7 b & $34,9 \mathrm{~b}$ & $1,7793 b$ & $40 a$ & $11 \mathrm{a}$ \\
\hline & Austrália & $26,4 \mathrm{~b}$ & $31,7 \mathrm{~b}$ & $1,5829 \mathrm{~b}$ & $36 a$ & $2 a$ \\
\hline \multirow{2}{*}{ E. benthamii } & $\mathrm{PCC} / \mathrm{SC}$ & $30,5 \mathrm{a}$ & $37,7 a$ & $1,9608 \mathrm{a}$ & $60 a$ & $11 \mathrm{a}$ \\
\hline & Austrália & $26,6 \mathrm{a}$ & $34,2 \mathrm{a}$ & $1,7388 \mathrm{a}$ & $51 \mathrm{a}$ & $20 \mathrm{~b}$ \\
\hline \multirow{3}{*}{ E. deanei } & $\mathrm{PCC} / \mathrm{SC}$ & $24,1 \mathrm{a}$ & $33,4 \mathrm{a}$ & $1,6886 \mathrm{a}$ & $62 \mathrm{a}$ & $24 a$ \\
\hline & Manville/SC & 23,6 a & $33,7 \mathrm{a}$ & $1,6831 \mathrm{a}$ & $76 a$ & $16 a$ \\
\hline & Klabin/PR & $21,9 \mathrm{a}$ & $30,9 \mathrm{a}$ & $1,5296 \mathrm{a}$ & $28 \mathrm{~b}$ & $8 a$ \\
\hline \multirow{3}{*}{$\begin{array}{c}E . \\
\text { macarthurii }\end{array}$} & Austrália & $21,7 a$ & $32,3 \mathrm{a}$ & $1,6169 \mathrm{a}$ & $61 \mathrm{a}$ & $10 a$ \\
\hline & Pieter Maritz & $20,9 a$ & $31,6 \mathrm{a}$ & $1,5721 \mathrm{a}$ & $51 \mathrm{~b}$ & $4 a$ \\
\hline & Wilsons Promontory & $20,5 \mathrm{a}$ & $30,6 \mathrm{a}$ & $1,5085 \mathrm{a}$ & $51 \mathrm{~b}$ & $1 \mathrm{a}$ \\
\hline \multirow{4}{*}{ E. viminalis } & Canela/RS & $21,3 \mathrm{~b}$ & $31,4 \mathrm{~b}$ & $1,5585 \mathrm{~b}$ & $26 \mathrm{~b}$ & $6 a$ \\
\hline & BGNDO & $18,2 b$ & 29,4 b & $1,4328 b$ & $31 \mathrm{~b}$ & $8 a$ \\
\hline & Nacional Campo & $21,9 \mathrm{~b}$ & $32,2 b$ & $1,6120 \mathrm{~b}$ & $37 a$ & $19 a$ \\
\hline & Manville/SC & 30,6 a & 36,9 a & $1,9136 \mathrm{a}$ & $41 \mathrm{a}$ & $12 a$ \\
\hline \multirow{4}{*}{ E. nitens } & Austrália & $19,7 \mathrm{a}$ & $30,0 \mathrm{a}$ & $1,4812 \mathrm{a}$ & $20,0 \mathrm{~b}$ & $18,8 \mathrm{a}$ \\
\hline & Glen Boc & $19,6 \mathrm{a}$ & $31,1 \mathrm{a}$ & $1,5406 \mathrm{a}$ & $40,0 \mathrm{a}$ & $3,1 \mathrm{a}$ \\
\hline & Macalista & $15,9 \mathrm{a}$ & $28,8 \mathrm{a}$ & $1,3857 \mathrm{a}$ & $17,5 \mathrm{~b}$ & $7,1 \mathrm{a}$ \\
\hline & Taboaganda & $15,4 a$ & $26,7 \mathrm{a}$ & $1,2625 \mathrm{a}$ & $20,0 \mathrm{~b}$ & $18,8 \mathrm{a}$ \\
\hline
\end{tabular}

*Médias na mesma coluna seguidas por letras distintas diferem entre si pelo teste de Scott-Knott a $P<0,05$. 
Para a estimativa dos parâmetros genéticos entre as procedências, as maiores herdabilidades $\left(h_{g}^{2}\right)$ para as variáveis dendrométricas (DAP, $\mathrm{H}$ e VOL) foram obtidas para $E$. viminalis, obtendo valores entre 0,2132 e 0,2314 (Tabela 4). Valores dessa herdabilidade na faixa de 0,20 a 0,30 têm sido observadas para a maioria dos caracteres quantitativos em espécies florestais (Resende, 2002), representando potencial de seleção. A espécie (E. viminalis) também apresentou os maiores valores para $V_{g}$ (variância genotípica entre procedências) e $A c_{\text {proc }}$ (Acurácia da seleção de procedências) reforçando que existe elevado potencial de seleção de procedências dentro da espécie.

De acordo com Fonseca et al. (2010), E. viminalis normalmente apresenta ampla variabilidade entre procedências, o que permite a seleção de genótipos superiores para o plantio. No presente estudo, dentre as procedências analisadas, pode-se destacar Manville/SC, a qual apresentou os melhores resultados para as variáveis quantitativas. Para as variáveis qualitativas não houve diferença significativa entre as procedências. De acordo com Konzen et al. (2017) a alta variabilidade genética dos indivíduos combinada com valores relativamente altos de herdabilidade pode implicar na previsão de ganhos genéticos consideráveis. Os autores destacam ainda que o ganho genético deve considerar a seleção de genótipos superiores e as melhores procedências.

Para E. smithii para os caracteres dendrométricos a $h_{g}^{2}$ ficou próxima a 0,08 (8\%), sendo a segunda espécie com maior valor de $h_{g}^{2}$. Para estes mesmos caracteres as outras espécies apresentaram valores de $h_{g}^{2}$ próximos ou abaixo de $5 \%$. Estes valores de herdabilidade representam baixo potencial de seleção das procedências. Em geral, herdabilidades individuais de baixa magnitude são comuns para caracteres quantitativos e, via de regra, conduzem a moderadas magnitudes das herdabilidades em nível de médias de progênies (Resende, 2002).

Estudo realizado por Konzen et al. (2017), revelou que procedências de E. smithii possuíam considerável variação genética para as variáveis diâmetro, altura, volume e retidão. Em geral, a maior parte da variação foi encontrada dentro das procedências. Em estudo na China realizado utilizando diversas procedências de E. smithii, em grande parte dos testes houve diferença entre procedências, demonstrando que a espécie apresenta grande variabilidade genética entre populações (Arnold et al., 2004).

Em relação à sobrevivência das plantas à campo, todas as espécies apresentaram diferenças entre procedências, com exceção de $E$. benthamii. A maior diferença entre procedências foi obtida para $E$. deanei, na qual as mudas originadas de sementes de Manville/SC apresentaram 76\% de sobrevivência, enquanto as provenientes da Klabin/PR obtiveram $28 \%$ de sobrevivência. Esse resultado demonstra como o local de origem das sementes exerce influência sobre a sobrevivência e desenvolvimento da espécie em novos locais, sendo que a procedência pode ser determinante para o sucesso de um empreendimento florestal. Em relação aos parâmetros genéticos, $E$. viminalis apresentou as maiores possibilidades de seleção, com maiores estimativas de $\mathrm{h}_{\mathrm{g}}^{2}$ e $A c_{\text {proc. }}$. No geral entre as espécies, a sobrevivência obteve parâmetros que expressam baixo potencial de seleção para a característica. Para bifurcação do fuste somente dentro de $E$. benthamii as procedências apresentaram diferença. Esta característica também não apresentou parâmetros genéticos que demostram potencial para seleção.

Para todos os caracteres a variância ambiental entre parcelas $\left(V_{\text {parc }}\right)$ apresentou valores relativamente altos comparando-se com os valores de variância fenotípica individual, para a maior parte das variáveis, indicando forte influência do ambiente sobre os genótipos. Geralmente, para parâmetros importantes como $V_{g}$ e $h_{g}^{2}$ entre os caracteres avaliados mostraram que não ocorreram variações significativas entre as procedências testadas, contudo pode-se ressaltar que há diferenças no desempenho entre as características e que a variabilidade existente pode permitir avanços expressivos em futuros programas de melhoramento genético de algumas espécies, especialmente para $E$. smithii e $E$. viminalis 
Tabela 4 - Resultado dos parâmetros genéticos para os caracteres diâmetro a altura do peito (DAP), altura $(\mathrm{H})$, volume $(\mathrm{VOL})$, sobrevivência (SOB) e bifurcação de procedências de Eucalyptus spp. em um plantio de 16 anos de idade em Rio Negrinho, SC. Brasil.

\begin{tabular}{|c|c|c|c|c|c|c|}
\hline Espécies & $\begin{array}{l}\text { Parâmetros } \\
\text { genéticos }\end{array}$ & DAP & $\mathbf{H}$ & VOL & SOB & BIF \\
\hline \multirow{8}{*}{ E. smithii } & $V_{g} *$ & 17,1445 & 3,7954 & 0,0114 & 0,0407 & 0,0017 \\
\hline & $V_{\text {parc }}$ & 19,4040 & 5,2448 & 0,0211 & 0,0287 & 0,0175 \\
\hline & $V_{e}$ & 135,8401 & 36,7028 & 0,1515 & 0,1441 & 0,1053 \\
\hline & $V_{f}$ & 172,4082 & 45,7447 & 0,1885 & 0,2285 & 0,1246 \\
\hline & $h_{g}^{2}$ & 0,0992 & 0,0835 & 0,0842 & 0,1045 & 0,0145 \\
\hline & $c^{2}$ parc & 0,1126 & 0,11464 & 0,1137 & 0,1256 & 0,1408 \\
\hline & $h_{m p}^{2}$ & 0,6962 & 0,6528 & 0,6414 & 0,3215 & 0,2080 \\
\hline & $A c_{\text {proc }}$ & 0,8345 & 0,8077 & 0,8084 & 0,5936 & 0,4561 \\
\hline \multirow{7}{*}{ E. benthamii } & $V_{g}$ & 1,0914 & 1,6315 & 0,0068 & 0,0201 & 0,0011 \\
\hline & $V_{\text {parc }}$ & 23,8014 & 9,7052 & 0,0398 & 0,0270 & 0,0278 \\
\hline & $V_{e}$ & 166,6442 & 67,9621 & 0,2785 & 0,2258 & 0,2047 \\
\hline & $V_{f}$ & 191,5425 & 79,3015 & 0,3250 & 0,2017 & 0,1048 \\
\hline & $h_{g}^{2}$ & 0,0065 & 0,02151 & 0,0208 & 0,0245 & 0,0189 \\
\hline & $c^{2}$ parc & 0,1242 & 0,12214 & 0,1224 & 0,1047 & 0,1494 \\
\hline & $h_{m p}^{2}$ & 0,1076 & 0,30545 & 0,3081 & 0,3078 & 0,2510 \\
\hline \multirow{10}{*}{ E. deanei } & $A c_{\text {proc }}$ & 0,3271 & 0,5535 & 0,5550 & 0,4632 & 0,4923 \\
\hline & $V_{g}$ & 0,0860 & 0,0825 & 0,0003 & 0,0148 & 0,0007 \\
\hline & $V_{\text {parc }}$ & 19,9940 & 6,6114 & 0,0276 & 0,0389 & 0,0247 \\
\hline & $V_{e}$ & 119,9642 & 39,6684 & 0,1624 & 0,1547 & 0,1487 \\
\hline & $V_{f}$ & 140,0445 & 46,3614 & 0,1891 & 0,2014 & 0,1743 \\
\hline & $h^{2} g$ & 0,0006 & 0,00185 & 0,0017 & 0,0412 & 0,0045 \\
\hline & $c^{2}$ parc & 0,1428 & 0,14266 & 0,1426 & 0,1289 & 0,1422 \\
\hline & $h_{m p}^{2}$ & 1,1595 & 3,2678 & 3,1791 & 0,2396 & 0,0783 \\
\hline & $A c_{\text {proc }}$ & 0,1077 & 0,1808 & 0,1783 & 0,3974 & 0,2798 \\
\hline & $V_{g}$ & 0,0524 & 0,0422 & 0,0027 & 0,0104 & 0,0013 \\
\hline \multirow{7}{*}{$\begin{array}{c}E . \\
\text { macarthurii }\end{array}$} & $V_{\text {parc }}$ & 13,3051 & 5,2497 & 0,0215 & 0,0175 & 0,0085 \\
\hline & $V_{e}$ & 79,8036 & 31,4981 & 0,1284 & 0,1783 & 0,0493 \\
\hline & $V_{f}$ & 93,1554 & 36,7918 & 0,1501 & 0,1984 & 0,0589 \\
\hline & $h_{g}^{2}$ & 0,0061 & 0,0011 & 0,0012 & 0,0176 & 0,0229 \\
\hline & $c^{2}$ parc & 0,1228 & 0,1421 & 0,1438 & 0,0987 & 0,1391 \\
\hline & $h_{m p}^{2}$ & 1,0487 & 2,135 & 2,1384 & 0,2178 & 0,3052 \\
\hline & $A c_{\text {proc }}$ & 0,1025 & 0,1467 & 0,1474 & 0,3874 & 0,5525 \\
\hline \multirow{3}{*}{ E. viminalis } & $V_{g}$ & 26,7745 & 8,2885 & 0,0346 & 0,0107 & 0,0002 \\
\hline & $V_{\text {parc }}$ & 10,5635 & 2,2985 & 0,0134 & 0,0178 & 0,0003 \\
\hline & $V_{e}$ & 88,1224 & 25,3185 & 0,1055 & 0,1078 & 0,095 \\
\hline
\end{tabular}


Tabela 4 - Continuação...

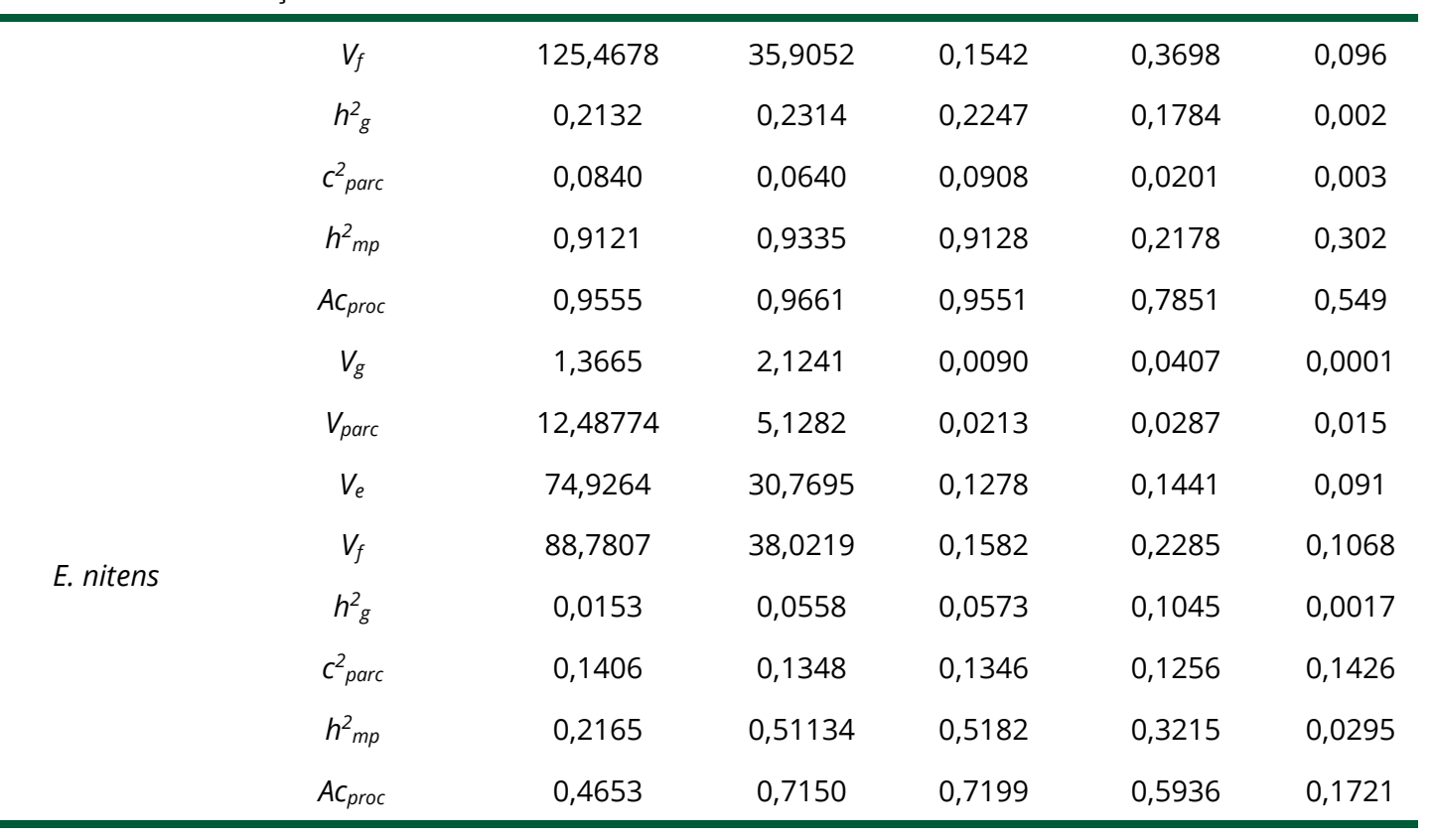

* Parâmetros genéticos: $V_{g}$ : variância genotípica entre procedências; $V_{\text {parc: }}$ variância ambiental entre parcelas; $V_{e}$ : variância residual dentro de parcela; $\mathrm{V}_{\mathrm{f}}$ : variância fenotípica individual; $\mathrm{h}^{2} \mathrm{~g}$ : herdabilidade individual no sentido amplo; $C^{2}$ parc: coeficiente de determinação dos efeitos da parcela; $h^{2}$ mp: herdabilidade da média da procedência; $A c$ proc: Acurácia da seleção de procedências.

De forma geral, pode-se dizer que pode haver outras espécies potenciais para o plantio no Sul do Brasil, como visto nos resultados do presente estudo, apresentando características qualitativas e quantitativas com potencial à seleção e multiplicação. Deve-se priorizar o uso de material genético adequado, considerando espécies e procedências, que entre outras características desejáveis, sejam tolerantes ao frio ou que apresentem boa capacidade de rebrota, possibilitando a regeneração das plantas em uma eventual ocorrência de geadas severas.

\section{CONCLUSÃO}

As espécies potenciais em relação às variáveis quantitativas foram E. smithii, E. dunnii, $E$. benthamii, E. badjensis e $E$. dorrigoensis. Já para as variáveis qualitativas pode-se destacar $E$. badjensis, E. macarthurii, E. benthamii e E. dorrigoensis, que apresentaram baixas taxas de mortalidade e de bifurcação, demonstrando boa adaptação nas condições ambientais locais.

Espécies como E. dunnii e E. benthamii já são plantadas comercialmente na região, porém, esse estudo mostra que outras espécies possuem potencial para serem exploradas, se adaptando ao frio, ocorrência de geadas, e apresentando elevada produtividade. Assim, são recomendados novos estudos principalmente com as espécies $E$. badjensis e E. dorrigoensis, que se destacaram nas variáveis qualitativas e quantitativas.

E. viminalis e E. smithii apresentaram os maiores valores para as estimativas dos parâmetros genéticos, demostrando que existo possibilidade de seleção dentro de suas procedências, principalmente para diâmetro, altura e volume.

\section{REFERÊNCIAS}

Aguiar, D., \& Mendonça, M. (2004). Climatologia das geadas em Santa Catarina. Simpósio Brasileiro de Desastres Naturais, 1, 762-773.

Alvares, C. A., Stape, J. L., Sentelhas, P. C., Gonçalves, J. L. M., \& Sparovek, G. (2013). Köppen's climate classification map for Brazil. Meteorologische Zeitschrift, 22(6), 711-728.

http://dx.doi.org/10.1127/0941-2948/2013/0507. 
Arnold, R. J., Clarke, B., \& Luo, J. (2004). Trials of cold-tolerant eucalypt species in cooler regions of south central China (Australian Center for International Agricultural Research Technical Reports, No. 57, pp. 106). Bruce: ACIAR.

Arnold, R., Li, B., Luo, J., Bai, F., \& Baker, T. (2015). Selection of cold-tolerant Eucalyptus species and provenances for inland frost-susceptible, humid subtropical regions of southern China. Australian Forestry, 78(3), 180-193. http://dx.doi.org/10.1080/00049158.2015.1063471.

Assis, T. F. (2014). Melhoramento genético de Eucalyptus: desafios e perspectivas (22 p.). Nova Lima: Embrapa Florestas.

Benin, C. C. (2014). Efeito do espaçamento na produção, variáveis dendrométricas e propriedades da madeira de Eucalyptus benthamii (Dissertação de mestrado). Universidade Estadual do Centro Oeste, Irati.

Brondani, E. G., Wendling, I., Grossi, F., Dutra, L. F., \& Araujo, M. A. (2010). Miniestaquia de Eucalyptus benthamii x Eucalyptus dunnii: (II) Sobrevivência e enraizamento de miniestacas em função das coletas e estações do ano. Ciência Florestal, 20(3), 453-465. http://dx.doi.org/10.5902/198050982060.

Carpanezzi, A. A., Pereira, J. C. D., Carvalho, P. E. R., Reis, A., Vieira, A. R. R., Rotta, E., Sturion, J. A., Rauen, M. J., \& Silveira, R. A. (1988). Zoneamento ecológico para plantios florestais no Estado de Santa Catarina (Documentos, No. 21, 113 p.). Curitiba: EMBRAPA-CNPF.

Chen, S. (2002). Cultivation and production of eucalypts in the People's Republic of China, with special reference to leaf oils. In J. J. W. Coppen (Ed.), The genus Eucalyptus (pp. 202-215). London: Taylor \& Francis. http://dx.doi.org/10.4324/9780203219430_chapter_8.

Correia, L. Q. (2011). Avaliação comparativa de SNPs e microssatélites para identificação individual, determinação de parentesco e ancestralidade em espécies de Eucalyptus (Dissertação de mestrado). Universidade Católica de Brasília, Brasília.

Darrow, W. K. (1994). Species trials of frost-tolerant eucalypts in the summer-rainfall zone of South Africa (pp. 8-12). Pietermaritzburg: Institute Commercial Forestry Research Newsletter.

Empresa de Pesquisa Agropecuária e Extensão Rural de Santa Catarina - EPAGRI. Centro de Informações de Recursos Ambientais e de Hidrometeorologia de Santa Catarina - CIRAM. (2009). Zoneamento Agroecológico. Florianópolis.

Ferreira, C. A., \& Couto, H. T. Z. (1981). A influência de variáveis ambientais no crescimento de espécies/procedências de Eucalyptus spp nos estados de Minas Gerais e Espírito Santo. Boletim de Pesquisa Florestal, (3), 9-35.

Fonseca, S. M., Resende, M. D. V., Alfenas, A. C., Guimarães, L. M. S., Assis, T. F., \& Grattapaglia, D. (2010). Manual prático de melhoramento genético do eucalipto Manual prático de melhoramento genético do eucalipto (200 p.). Viçosa: UFV.

Food and Agriculture Organization - FAO. (1981). El eucalipto en la repoblacion forestal (723 p.). Roma.

Frigotto, T., Navroski, M. C., Nicoletti, M. F., Felippe, D., Novak Junior, N. S., Galvani, L. V., \& Souza, P. F. (2015). Equações volumétricas para povoamento clonal de Eucalyptus sp. no Norte de Santa Catarina. In Anais do III Simpósio Internacional de Ciência, Saúde e Território (Vol. 1, pp. 227-2237). Lages: Universidade de Planalto Catarinense.

Hesheng, L., Jingqing, Q., Guoyang, X., Delong, H., Shirong, X., \& Wenge, L. (2003). Review of cold-tolerant Eucalyptus improvement in Fujian Province. In J. W. Turnbull (Ed.), Eucalypts in Asia: Proceedings of an International Conference Held in Zhanjiang, Guangdong, People's Republic of China (ACIAR Proceedings, No. 111, pp. 117-122). Canberra: ACIAR.

Higa, A. R., \& Higa, R. C. V. (2000). Indicação de espécies para reflorestamento. In Embrapa Comunicação para Transferência de Tecnologia (Ed.), Reflorestamento de propriedades rurais para fins produtivos e ambientais (1. ed. Cap. 6, pp. 101-124). Brasília: EMBRAPA/CTT.

Higa, R. C. V., Higa, A. R., \& Alves, E. C. (2002). Comportamento de progênies de Eucalyptus badjensis Beuzev. \& Welch em dois locais da Região Sul do Brasil. Boletim Pesquisa Florestal, 45, 89-97.

Higa, R. C. V., Higa, A. R., Trevisan, R., \& Souza, M. V. R. (1997). Comportamento de vinte espécies de Eucalyptus em área de ocorrência de geadas na região Sul do Brasil. In Anais da Conferência IUFRO sobre Silvicultura e Melhoramento de Eucaliptos (Vol. 1, pp. 106-110). Colombo: EMBRAPA-CNPF.

Indústria Brasileira de Árvores - IBÁ. (2017). Relatório 2017 (80p.). Brasília.

Kageyama, P. Y., Vencovsky, R., Ferreira, M., \& Nicolielo, N. (1977). Variação genética entre procedências de Pinus oocarpa Schiede na região de Agudos - SP. IPEF, (14), 77-120. 
Kobiyama, M., Fruet, D., Lima, R. T., Marschne, M., Guimarães, L. M. R., \& Chaffe, P. L. B. (2004). Estudo hidrológico comparativo entre três pequenas bacias experimentais no município de Rio Negrinho SC: descrição. In Anais do V Simpósio Nacional de Geomorfologia e I Encontro Sul-Americano de Geomorfologia. Santa Maria: UFSM.

Konzen, E. R., Navroski, M. C., Pereira, M. O., Nascimento, B., Meneguzzi, A., \& Souza, P. F. (2017). Genetic variation for growth variables of Eucalyptus benthamii Maiden \& Cambage and E. smithii R. T. Baker provenances in Southern Brazil. Cerne, 23(3), 359-366. http://dx.doi.org/10.1590/01047760201723032357.

Lisbão Junior, L. (1980). O efeito da geada e o comportamento inicial de três procedências de Eucalyptus dunnii Maiden, em ensaio conjugado de miniespaçamentos e adubação. Boletim de Pesquisa Florestal, (1), 28-49. http://dx.doi.org/10.1590/01047760201723032357.

Moura, V. P. G. (2001). Potencial e uso de espécies de Eucalyptus e Corymbia de acordo com locais e usos (Documentos, No. 68, 32 p.). Brasilia: Embrapa.

Mujiu, L., Arnol, R. J., Bohai, L., \& Minsheng, Y. (2003). Selection of cold-tolerant eucalypts for Hunan province. In J. W. Turnbull (Ed.), Eucalypts in Asia: Proceedings of an International Conference Held in Zhanjiang, Guangdong, People's Republic of China (ACIAR Proceedings, No. 111, pp. 107-116). Canberra: ACIAR.

Müller, B. V., Rocha, M. P., Klitzke, R. J., Silva, J. R. M., \& Cunha, A. B. (2017). Produção de madeira serrada com cinco espécies de eucalipto resistentes à geada. Advances in Forestry Science, 4(4), 195-201.

Paludzyszyn Filho, E., \& Santos, P. E. T. (2011). Programa de melhoramento genético de eucalipto da Embrapa Florestas: resultados e perspectivas (Documentos, No. 214). Colombo: Embrapa.

Paludzyszyn Filho, E., Santos, P. E. T., \& Ferreira, C. A. (2006). Eucaliptos indicados para plantios no estado do Paraná (Documentos, No. 129, 45 p. ). Colombo: Embrapa Florestas.

Quiqui, E. M., Martins, S. S., \& Shimizu, J. Y. (2001). Avaliação de espécies e procedências de Eucalyptus para o Noroeste do Estado do Paraná. Acta Scientiarum, 23(5), 1173-1177.

Resende, M. D. V. (2002). Genética biométrica e estatística no melhoramento de plantas perenes (975 p.). Brasília: Embrapa Informação Tecnológica.

Resende, M. D. V. (2006). O software Selegen-Reml/Blup. Campo Grande: Documentos EMBRAPA.

Santos, Á. F., Auer, C. G., \& Grigoletti Junior, A. (2001). Doenças do eucalipto no sul do Brasil: identificação e controle (Circular Técnica, No. 45, pp. 1-20). Colombo: Embrapa.

Swain, T. L., \& Gardner, W. A. (2003). Use of site-species matching and genetic gain to maximize yield: a South African example. In R. P. Wei \& D. Xu (Eds.), Eucalypt Plantations: Research, Management and Development. Proceedings of an International Symposium (pp. 167-185). Singapore: World Scientific.

Wrege, M. S., Steinmetz, S., Reisser Junior, C., \& Almeida, I. R. (2012). Atlas climático da região sul do Brasil: Estados do Paraná, Santa Catarina e Rio Grande do Sul (2. ed., 334 p.). Brasília: EMBRAPA.

Yi, Y., Youbing, W., Yinzhong, E., Qinghong, Z., \& Yating, W. (2014). Analysis of the freezing damage to biennial Eucalyptus dunnii and Eucalyptus smithii in Haikou Forest Farm. Meteorological and Environmental Research, 5(8), 31-38.

Zhang, R., Sasse, J., Arnold, R., \& Kube, P. (2003). Tree improvement plan for E. smithii in Yunnan (Vol. 7). Victoria: Forest Science Centre Report.

Contribuição dos autores: TF: investigação, metodologia, supervisão, software, escrita - primeira redação; MCN: conceituação, curadoria de dados, obtenção de financiamento, metodologia, supervisão, escrita - revisão e edição; NSA: validação, escrita - revisão e edição; DF: investigação, visualização, software; GAB: conceituação, obtenção de financiamento, administração do projeto; MOP: validação, visualização, escrita - primeira redação; QCL: visualização, escrita - primeira redação. 